\title{
Does tight glycemic control improve outcomes in pediatric patients undergoing surgery and/or those with critical illness?
}

This article was published in the following Dove Press journal:

International Journal of General Medicine

4 December 2013

Number of times this article has been viewed

\author{
Neil Christopher Forbes \\ Nicola Anders \\ Department of Anaesthesia, Royal \\ Manchester Children's Hospital, \\ Greater Manchester, England
}

Correspondence: Neil Christopher Forbes

Manchester Royal Infirmary, Oxford Road, Manchester, Greater Manchester, MI3 9WL, UK

Emailncforbes@doctors.org.uk

\begin{abstract}
This literature review examines the current evidence regarding the potential usefulness of tight glycemic control in pediatric surgical patients. In adults, fluctuations in glucose levels and/or prolonged hyperglycemia have been shown to be associated with poor outcomes with respect to morbidity and mortality. This review begins by summarizing the findings of key papers in adult patients and continues by investigating whether or not similar results have been seen in pediatric patients by performing a comprehensive literature review using Medline (OVID). A database search using the OVID interface and including the search terms (exp glucose) AND (exp surgery) AND (exp Paediatric/pediatric) AND (exp Hypoglycaemia/hypoglycemia) AND (exp Hyperglycaemia/hyperglycemia) yielded a total of $150+$ papers, of which 24 fulfilled our criteria. We isolated papers utilizing pediatric patients who were hospitalized due to illness and/or surgery. Our review highlights several difficulties encountered in addressing this potentially useful clinical intervention. An absence of scientifically robust and randomized trials and the existence of several small-powered trials yielding conflicting results mean we cannot recommend tight glycemic control in these patients. Differences in study design and disagreements concerning the crucial stage of surgery where hyperglycemia becomes important are compounded by an over-reliance on the discretion of clinicians in the absence of well described treatment protocols. Closer inspection of key papers in adult patients identified fundamental discrepancies between exact definitions of both hyperglycemia and hypoglycemia. This lack of consensus, along with a fear of inducing iatrogenic hypoglycemia in pediatric patients, has resulted in professional bodies advising against this form of intervention. In conclusion, we cannot recommend use of tight glycemic control in pediatric surgical patients due to unclear glucose definitions, unclear thresholds for treatment, and the unknown long-term effects of iatrogenic hypoglycemia on the developing body and brain.
\end{abstract}

Keywords: pediatric, hypoglycemia, hyperglycemia, insulin, surgery, glucose

\section{Introduction}

Surgery and/or critical illness can result in an increase in blood glucose levels, known as hyperglycemia. ${ }^{1}$ This "stress diabetes" or "diabetes of injury" occurs due to relative insulin resistance and an increase in hepatic glucose production. ${ }^{2}$ It was widely thought that this surge in blood glucose provided a readily accessible energy source and formed part of the evolutionary "fight or flight" response. ${ }^{1}$ It has become increasingly apparent that what was initially presumed to be a "natural" and "protective" response may be a deleterious process worthy of clinical prevention and intervention. ${ }^{3}$ Several studies have shown an association between hyperglycemia and poor morbidity and mortality, irrespective of pathology, in adult patients. ${ }^{1,2,4}$ However, the relationship 
between blood glucose and secondary outcomes has not been clearly defined, with studies showing mixed results. ${ }^{5}$ Positive results in adult patients when intensive insulin therapy was used have led to increased interest as to whether this method of intervention may be applicable to a pediatric population who are most susceptible to the potentially damaging effect of fluctuating or unacceptably high/low blood glucose levels due to their physiologic immaturity. ${ }^{6}$ This paper evaluates the evidence for and against tight glycemic control in children and critically evaluates key papers using adult studies which provide the basis of subsequent investigation in this target group.

\section{Pathophysiology: the deleterious effects of glucose}

The characteristic increase in blood glucose levels is a result of counter-regulatory hormones, including glucagon, growth hormone, catecholamines, and cytokines, such as interleukin-1, interleukin-6, and tumor necrosis factor-alpha, that result in increased gluconeogenesis and glycogenolysis. ${ }^{7,8}$ In principle, once the stressor(s) has been removed, euglycemia should be achieved by the action of insulin, which facilitates glucose entry into insulin-sensitive glucose channels. ${ }^{8}$ Many studies in adults have shown that the duration and intensity of hyperglycemia are directly related to postoperative outcomes, and it is thought that this is due to prolonged exposure to a stressor, in this case invasive surgery. ${ }^{8}$ In essence, the longer an individual is exposed to an external stressor such as the trauma of surgery or prolonged critical illness, ${ }^{8}$ the more likely it is for insulin resistance to develop due to the effect of insulin-like growth factorbinding protein-1. ${ }^{1}$ High glucose levels affect a number of other aspects of physiology, some of which are hypothesized to play a role in postoperative outcomes, including oxidative injury, a proinflammatory response, clotting abnormalities, vascular reactivity, and decreased immune system effectiveness. ${ }^{7}$ It is thought that hyperglycemia compromises all major components of the innate immune system, including phagocytosis and opsonization, ${ }^{9}$ causing glycosylation and inactivation of circulating immunoglobulins that contributes to an increased risk of infection and is an important cause of postoperative mortality in vulnerable individuals. ${ }^{2}$ Importantly, coagulopathies and hyperglycemia tend to occur simultaneously and correlate with the magnitude of trauma and wound size. 9,10 $^{9}$

Studies utilizing intensive insulin therapy have shown a reduction in circulating markers of inflammation, such as C-reactive protein and mannose-binding lectin. ${ }^{2}$
Further, a prolonged hospital stay in an intensive care unit (ICU) is associated with a hypercatabolic state and a feedingresistant wasting syndrome that can be ameliorated by the stimulatory effect of insulin on muscle protein synthesis and its ability to reduce the breakdown of protein. ${ }^{2}$ Overall, by preventing hyperglycemia with insulin, it is thought that bioenergetic failure can be prevented and this could protect organs from glucose-related damage. ${ }^{2}$ Generally speaking, studies investigating intensive insulin therapy have tended to focus on diabetic patients because these patients form a unique group in which blood glucose control is of paramount importance; however, the insulin resistance and subsequent hyperglycemia described here is often seen in individuals who have never had diabetes, suggesting that interventions may have a very wide scope of effectiveness. ${ }^{4,11,12}$ Many studies evaluating the potential usefulness of intensive insulin therapy in both adults and children have enrolled patients undergoing cardiac surgery due to the effect of glucose on the heart and the ease of glucose sampling. ${ }^{8}$ Hyperglycemia alters cardioprotective signal transduction pathways, increases myocardial infarction size, and alters the microcirculatory system of the heart; further, animal studies have shown increased systemic vascular resistance, decreased stroke volume, and impaired cardiac output. ${ }^{8}$ Another organ particularly susceptible to the damaging effects of hyperglycemia is the kidney, where it is thought that hyperglycemia may induce renal mesangial cell apoptosis and increase the risk of acute kidney injury and renal failure. ${ }^{8}$ It is unclear how intensive insulin therapy works, but insulin functions through the actions of two intracellular pathways, ie, the "mitogenic" pathway via Shc/Grb2 activation, leading to activation of mitogen-activated protein kinase isoforms, and the "metabolic" pathway that proceeds via insulin receptor substrates in a manner dependent on activation of phosphoinositide 3 -kinase. ${ }^{2}$ It is thought that in critically ill patients, activation of the metabolic pathway and an increase in messenger (m)RNA encoding glucose transporter type 4 receptor and hexokinase II, which is a ratelimiting enzyme in glucose metabolism, increases glucose uptake in skeletal muscle. $^{2}$

\section{Intensive insulin therapy in adult patients}

It is widely accepted that a guidance range for normoglycemia is $90-140 \mathrm{mg} / \mathrm{dL}$, and that in coronary syndromes, a lower target range of $80-100 \mathrm{mg} / \mathrm{dL}$ should be used to reduce mortality. ${ }^{5}$ A level $<180 \mathrm{mg} / \mathrm{dL}$ leads to better outcomes in adults, but the threshold for children is less clear-cut. ${ }^{13}$ Standard treatment is to start insulin once blood 
glucose levels exceed $215 \mathrm{mg} / \mathrm{dL}$, defined as the "renal threshold" after which glycosuria and hypovolemia may occur, although this can occur at lower values between $125 \mathrm{mg} / \mathrm{dL}$ and $250 \mathrm{mg} / \mathrm{dL} .^{5,13}$ In 2001, Van den Berghe et al published the results of a prospective, randomized study investigating the role of intensive insulin therapy in critically ill patients which promised a great deal. ${ }^{4} \mathrm{~A}$ total of 1,548 patients were enrolled over a period of 12 months. ${ }^{4}$ Patients were split into two groups, ie, a treatment arm with target blood glucose levels of $80-100 \mathrm{mg} / \mathrm{dL}$ and a standard treatment arm with target blood glucose levels of $180-215 \mathrm{mg} / \mathrm{dL} .^{8}$ This was important because standard treatment prior to publication of this trial had been to intervene only when levels had reached $220 \mathrm{mg} / \mathrm{dL}$ and to maintain levels at $180 \mathrm{mg} / \mathrm{dL}$ with treatment. ${ }^{2}$ The authors hypothesized that normalization of blood glucose levels in critically ill patients could reduce mortality and morbidity, the risk of which is thought to be $20 \%$ in those requiring more than 5 days in an ICU. ${ }^{4}$ The results showed that intensive insulin therapy reduced ICU mortality by $3.4 \%$ and overall hospital mortality by $3.7 \%$ in critically ill patients admitted to a surgical ICU. ${ }^{8}$ From an economic viewpoint, the intervention also led to a decrease in requirement for ICU resources and a reduction in use of long-term antibiotics due to a lower infection rate in the treatment arm. ${ }^{8}$ This study provided a platform suggesting that regulation of blood glucose levels in an ICU could have wide-reaching implications for clinical practice. ${ }^{2,8}$ However, it was not known whether the degree of hyperglycemia contributed directly to pathology or whether it served as a marker. ${ }^{2}$ Nevertheless, by manipulating a process previously thought to be "natural", it seemed possible to significantly improve outcomes in this patient group and subsequent studies were carried out.

Traumatic brain injury is an example of an insult that can have severe and profound effects in a relatively short space of time; further, the lasting effects of neurologic injury can be considerable. In 2009, Meng et al studied the use of intensive insulin therapy in a cohort of 240 adult patients (mean age 46 years), 121 of whom were allocated to a treatment group to reach glucose levels of $80-110 \mathrm{mg} / \mathrm{dL}$ and 119 patients received "conventional" treatment, ie, only treated if levels exceeded $200 \mathrm{mg} / \mathrm{dL} .{ }^{14}$ The primary outcome of the study was mortality at 6 months and secondary outcomes consisted of ICU infection, duration of ICU stay, inhospital mortality, and neurologic follow-up at 6 months. ${ }^{14}$ Importantly, the authors included a statistical analysis of the impact of age and sex on 6-month follow-up in an attempt to define any bias that may have been apparent, but no difference was seen $(P=0.8) .{ }^{14}$ Infection rates were higher in the group receiving conventional treatment compared with intensive insulin therapy ( $46.2 \%$ versus $31.4 \%, P<0.05)$, days spent in an ICU was shorter in the intensive insulin therapy group (4.2 days versus 5.6 days [median], $P<0.05)$, and inhospital mortality did not differ between the groups $(28.6 \%$ versus $28.9 \%$, $P=0.85) .{ }^{14}$ Importantly, neurologic outcome was better in the intensive insulin therapy group at 6 months $(29.1 \%$ versus $22.4 \%, P<0.05)$ suggesting that intensive insulin therapy may have long-term benefit in this patient group. ${ }^{14}$

Limitations of the study included the exclusive enrollment of the most severe traumatic brain injuries, patients with a Glasgow Coma Score of 3-8, and that overall the primary insult is the most defining characteristic in these patients. ${ }^{14}$ The authors found no significant difference in the incidence of hypoglycemic episodes and used this as the rationale for advocating intensive insulin therapy but opted to use a cutoff of $<40 \mathrm{mg} / \mathrm{dL}$, similar to Van den Berghe et al, ${ }^{4}$ instead of $<80 \mathrm{mg} / \mathrm{dL}$ as used in other studies, which has the potential to miss hypoglycemic episodes. ${ }^{14}$ This study suggested that intensive insulin therapy could reduce intracranial pressure while using significantly less concentrations of vasopressors, and that by 12 months, a larger fraction of survivors would be rehabilitated to independent living. ${ }^{14}$

In 2010, Marik and Preiser conducted a meta-analysis of randomized controlled trials (RCTs) concerning the usefulness of intensive insulin therapy in adult patients. ${ }^{7}$ In seven RCTs, the main finding was that outcomes may be directly affected by the method of nutrition received by the patients; those receiving a high parenteral glucose load may benefit from intensive insulin therapy whereas those receiving enteral nutrition may come to harm if intensive insulin therapy was used. ${ }^{7}$ The authors suggested that this could be due to the effect of oral glucose on glucose-dependent insulinotropic polypeptide and glucagon-like peptide-1, which activate incretin receptors on pancreatic beta-cells and increase intracellular cyclic adenosine monophosphate (cAMP) and calcium, leading to exocytosis of insulin. ${ }^{7}$ Further, peripheral glucose administration is likely to increase hepatic glucose output. ${ }^{7}$ It is therefore thought that parenteral nutrition creates a significantly higher blood glucose level which causes cellular glucose overload and oxidative injury in cells that do not require insulin for glucose uptake. ${ }^{7}$ Another small study recommended a reduction in glucose infusion as opposed to the aggressive use of insulin. ${ }^{15}$ Gandhi et al quantified the risk of intraoperative glucose and adverse events by formulating a continuous scale whereby every $20 \mathrm{mg} / \mathrm{dL}$ increase in blood glucose above $100 \mathrm{mg} / \mathrm{dL}$ was associated with a $34 \%$ increase in the likelihood of an adverse event. ${ }^{16}$ 
In 2012, Hua et al conducted a meta-analysis of studies using intensive insulin therapy against conventional therapy during cardiac surgery. ${ }^{17}$ Five RCTs were identified, comprising 706 patients. ${ }^{17}$ The results showed that the overall risk of 30-day inhospital mortality was insignificant between the two groups $(P=0.25) .{ }^{17}$ Further, the risk of hypoglycemia events was also insignificant $(P=0.26)$, but the risk of infection was significantly lower in the intensive insulin therapy group $(P=0.009) .{ }^{17}$ One drawback of this particular analysis was the low incidence of deaths $(n=10)$, and as such, it could be argued that the insignificant results seen in mortality may be due to chance. ${ }^{17}$ Overall, the authors concluded that due to variability in patients and the procedures carried out, the data were inconsistent, but suggested that, in certain patient groups, notably diabetic patients undergoing cardiac surgery and requiring cardiopulmonary bypass, tight glucose control could be crucial. ${ }^{17}$ There were several other limitations of this study which the authors remark could account for the results seen, including variability in glucose monitoring and management and enrollment of both diabetic and nondiabetic patients, with no way of distinguishing between the two groups. ${ }^{17}$ Overall, the authors called for large-scale randomized trials to be carried out to ascertain whether any benefit could be seen.

The outcomes of several other trials seemed to suggest that the initial findings of Van den Berghe et al may have been irreproducible, so Van den Berghe et al performed a systematic review of all published RCTs on glucose control in the ICU to ascertain why the outcomes had been so different to their initial study. ${ }^{5}$ They concluded that several differences accounted for the heterogeneity between their paper and others, including: study design, different intervention groups, routes of insulin administration, types of infusion pumps used, sampling sites, accuracies of glucose meters, nutritional strategies, and varying expertise, all of which mean it is not possible to recommend a single optimal glucose target in the ICU. ${ }^{5}$ Generally speaking, they recommended age-adjusted levels of glycemia for use in units that may not be equipped or have staff with adequate expertise in administering intravenous insulin. ${ }^{5}$ Van den Berghe et al argue that their study was particularly robust due to the use of continuous insulin infusion via a central line using a syringe-driven insulin pump, which is thought to be especially accurate..$^{5}$ They also remarked that insulin dose calculations were carried out by nurses with significant expertise in performing high-level decision-making. ${ }^{5}$ Reductions in ICU and hospital mortality seen in a surgical setting were not significant when tested in a medical ICU setting. ${ }^{5}$ Van den Berghe et al suggest that in the medical setting, organ damage had already occurred prior to intervention, which meant a lack of opportunity to prevent this by lowering glucose which could be the case if this is indeed the mechanism of action of intensive insulin therapy. ${ }^{5}$ Unfortunately, we do not know whether glucose forms part of a pathology or is a marker of some other as-yet undefined process. In a rather defensive critique of other studies, Van den Berghe et al concluded that one common denominator links all other trials attempting to reach the same outcomes as their pioneering study, and that is they are "... all statistically underpowered to detect a reasonable mortality difference". 5

One such trial was NICE-SUGAR (Normoglycemia in Intensive Care Evaluation-Survival Using Glucose Algorithm Regulation) which enrolled 6,100 patients and aimed to see whether intensive insulin therapy could induce a $3.8 \%$ reduction in mortality from a baseline of $30 \% .{ }^{5}$ Van den Berghe et al state that due to discrepancies in definitions of hypoglycemia and hyperglycemia, it could be argued that the NICE-SUGAR trial was destined to fail since glucose definitions were closer together than those used in the Leuven Intensive Insulin trial. ${ }^{5}$ Overall, the NICE-SUGAR trial suggested that intensive glucose control increased mortality among adults in the ICU and that a blood glucose target of $180 \mathrm{mg} / \mathrm{dL}$ or less resulted in lower mortality than did a target of $80-108 \mathrm{mg} / \mathrm{dL} .{ }^{18}$ Importantly, no significant difference was seen between two treatment groups with regard to median number of days in the ICU $(P=0.84)$ or hospital $(P=0.86)$, median number of days of renal replacement therapy $(P=0.39)$, or days of mechanical ventilation $(P=0.56) .{ }^{18}$ The 90 -day mortality was reduced from $27.5 \%$ to $24.9 \%$ if a target of $<108 \mathrm{mg} / \mathrm{dL}$ was used. ${ }^{18}$ Since this study seemed to refute the findings of Van den Berghe et al that a level of $80-100 \mathrm{mg} / \mathrm{dL}$ should be advocated, Van den Berghe et al responded in their critique.

The use of a definition of normoglycemia at $140-180 \mathrm{mg} / \mathrm{dL}$ instead of $180-215 \mathrm{mg} / \mathrm{dL}$, Van den Berghe et al argued, made the two studies fundamentally different. ${ }^{5}$ An important point since the Van den Berghe et al trial utilized existing practices that glucose levels below $215 \mathrm{mg} /$ $\mathrm{dL}$ should not be treated, in contrast with $70 \%$ of treated patients in the NICE-SUGAR trial who had an intermediate glucose level of 140-180 mg/dL. ${ }^{5,18}$ Nevertheless, even if those patients would not have necessarily been treated in the Leuven trial, Van den Berghe et al agree that the outcomes of the NICE-SUGAR trial suggest that this could be a useful set of values to aim for in the ICU. ${ }^{5}$ Another criticism was the lack of glucose maintenance in patients in the NICE-SUGAR 
trial which was below $50 \%$ compared with $70 \%$ in the Leuven study and could account for the disappointing results seen in subsequent trials. ${ }^{5}$ Van den Berghe et al argue that this is not a simple intervention but one that requires significant expertise and consistency in glucose monitors, without which episodes of hypoglycemia could pass under the radar. ${ }^{5}$ In what could be an important consideration, Van den Berghe et al state that potassium levels were monitored during their study because of the effect of insulin on potassium levels and the risk of arrhythmias, and the lack of such measurement in other studies could have predisposed participants to complications and even death due to the secondary effects of insulin which were not documented. ${ }^{5}$ To conclude, both studies agree that glucose is important during critical illness because fluctuations both ways affect outcome in both directions but a consensus on optimal glucose levels still evades our best efforts. ${ }^{5}$ Van den Berghe et al concluded their analysis by recommending a "fall-back" approach of avoiding fluctuations and/or prolonged periods of either hypoglycemia or hyperglycemia..$^{5,11}$

It may be that fluctuations in blood glucose levels are the real culprit and that patients may be able to tolerate prolonged episodes of mild hyperglycemia. ${ }^{5}$ The problem is that we do not know exactly what level we can consider to be "normal" and at what exact level we are putting patients at risk. It is important to assess the benefits versus the risk of iatrogenic hypoglycemia, an especially important consideration in pediatric patients. A study by De La Rosa et al showed no benefit of intensive insulin therapy on morbidity and mortality in a mixed ICU, but showed a statistically significant increase in hypoglycemic episodes $(17 \%$ versus $4.1 \%, P<0.001) .{ }^{12}$

\section{Intensive insulin therapy in pediatric patients}

Pediatric intensive care units regularly report hyperglycemia in their patients; an estimated $80 \%$ have levels $>110 \mathrm{mg} / \mathrm{dL}$, $60 \%$ have levels $>150 \mathrm{mg} / \mathrm{dL}$, and $30 \%$ have levels $>200 \mathrm{mg} /$ $\mathrm{dL}$, according to Vlasselaers et al. ${ }^{19}$ Further, the duration of hyperglycemia correlates with prognosis and outcomes. ${ }^{19}$ It is therefore perhaps unsurprising that given the promising data advocating intensive insulin therapy in adults, that researchers aimed to see if the findings could be extrapolated to a pediatric population. Vlasselaers et al conducted a prospective RCT in 317 infants (under 1 year of age) and 383 children (from under 1 year of age through to 16 years) admitted to the pediatric intensive care unit in a single center, $75 \%$ of whom had undergone cardiac surgery. ${ }^{19}$ The group randomized to intensive insulin therapy were treated to reach a blood glucose level of 50-80 mg/dL and those randomized to conventional treatment were treated to achieve a blood glucose level of 70-100 mg/dL. ${ }^{19}$ Mean blood glucose levels were reduced to a statistically significant level in both infants and children, with those receiving intensive insulin therapy reaching the lowest $(P<0.001)$, but hypoglycemia occurred at a higher rate in $25 \%(n=87)$ of the intensive insulin therapy group compared with $1 \%(n=5)$ in the conventional treatment group. ${ }^{19}$ An important finding was a shorter duration of ICU stay in the intensive insulin therapy group (5.51 days versus 6.15 days, $P=0.017$ ), and $38 \%$ of the intensive insulin therapy group required an extended ICU stay (more than the median) compared with $47 \%$ in the conventional treatment group $(P=0.013) .{ }^{19}$ Mortality was lower in the intensive insulin therapy group to a statistically significant level (3\% versus $6 \%, P=0.038) .{ }^{19}$ Unfortunately, the number needed to treat to derive benefit from strict glycemic control was higher than the number needed to harm due to hypoglycemia. ${ }^{19}$ Overall, this study emphasized the problems with using intensive insulin therapy in pediatric patients, where for some patients it could be extremely beneficial but at the risk of causing significant harm in others. ${ }^{19}$

This study has several positive and negative points to its design. One area of concern is the fact that some patients were excluded on the basis of "... a medical disorder that the treating physician regarded as unsuitable", with no explanation of why this was the case and/or which conditions were omitted. This represents an especially important omission since the benefit of intensive insulin therapy has sometimes only been seen in individuals with particular conditions, eg, diabetes. ${ }^{19}$ One major problem with studies of this nature has been the lack of a predetermined protocol for the treatment of blood glucose levels. Vlasselaers et al used an age-adjusted normal fasting glucose concentration as a guide for the use of an insulin infusion throughout care; an important point, since other studies investigating pediatric patients enrolled children aged $0-18$ years and gave no information regarding a formal approach to managing glucose levels other than simply ".. at the discretion of the physician". ${ }^{19}$ The authors note that we cannot predict the long-term effects of hypoglycemic episodes on a child's development, ${ }^{19}$ and as such, any intervention, even if it shows short-term gain, cannot be universally adopted until long-term, standardized trials are carried out. This study utilized several markers of damage and showed that in children undergoing cardiac surgery, intensive insulin therapy can reduce biomarkers of heart damage, including troponins and heart-type fatty acid binding protein. ${ }^{19}$ Further, lactate concentrations remained 
within range in the intensive insulin therapy group, suggesting reduced glycolysis or improved mitochondrial function, both of which are important considerations due to the actions of insulin once relative insulin resistance has formed. ${ }^{19}$ Importantly, it is thought that organ damage occurs due to reperfusion injury after surgery and this could potentially be curtailed by insulin therapy. ${ }^{19}$ Despite the high rate of hypoglycemic episodes in the treatment group, Vlasselaers et al argue that even if an episode occurs, fast action and management could reduce the risk of any long-term effects because it is rebound hyperglycemia and not necessarily hypoglycemia per se that causes neuronal damage. ${ }^{19}$

A crucial point in the significant outcomes of this study was the use of an age-adjusted measurement for blood glucose, and the authors themselves concede that if the glucose concentrations were comparable with that of adults, the results would have been insignificant, a finding which echoes that of Van den Berghe et al. ${ }^{5,19}$ Further, despite seeming like a positive result, the authors note the considerable expertise required by nursing staff for this to be not only effective but also safe, and this has implications for cost and staffing. ${ }^{19}$ However, these promising results were not replicated by Agus et al, who investigated the effect of tight glycemic control in 444 children and found that although normoglycemia was achieved sooner and was maintained for longer by using intensive insulin therapy $(P<0.001)$, this had no effect on secondary outcomes, including infection, and did not benefit high-risk groups. ${ }^{1}$

\section{Standards of care}

A survey carried out by Hirshberg et al aimed to elucidate current standards of practice among adult and pediatric intensivists. ${ }^{20}$ Their results highlighted a great deal of variation between optimal glucose ranges and exact definitions of both hypoglycemia and hyperglycemia. ${ }^{20}$ Algorithms for the initiation and maintenance of insulin therapy differed widely between doctors, emphasizing the need for large-scale trials to generate accepted standards of practice. ${ }^{20}$ Interestingly, the survey showed that clinicians tend to undertreat due to a fear of inducing iatrogenic hypoglycemia. This was especially the case with the pediatric intensivists because of the unknown long-term effects of hypoglycemia. ${ }^{15,20}$ As documented in recently published correspondence, opinion still differs on what are considered "correct" blood glucose levels to aim for. ${ }^{20}$ To address this issue, Steil et al performed a study to find out whether using a continuous glucose monitor would be a cost-effective and more scientifically robust method of measuring glucose levels. ${ }^{21}$ Unfortunately, the results showed that the technology required does not yet exist because a lack of consistency in cutoff values and the susceptibility to false alarms or failure to recognize hypoglycemia led to an increase in workload for nursing staff, the opposite of what was hoped for. ${ }^{22}$ Nevertheless, if technology were able to increase its efficiency, it could be a realistic opportunity for the future. Piper et al also investigated the usefulness of real-time glucose monitors and concluded that, while they have a place in therapy, the interruption of signals occurred regularly in theater. ${ }^{23}$ One major drawback of using a subcutaneous glucose monitor is the difference between interstitial and blood glucose; these may be quite different, especially in conditions where perfusion of subcutaneous tissue is compromised. ${ }^{23}$

\section{Pediatric hyperglycemia}

Hyperglycemia can occur theoretically at any point during an adult's or child's stay in hospital, but intraoperative hyperglycemia has been shown to be associated with a higher risk of postoperative infections in adult patients undergoing cardiac surgery. ${ }^{24}$ Monitoring of blood glucose during surgery is especially important, given that even a short period can induce upregulation of proinflammatory factors, and this effect can last for up to 6 days, which is a great deal of time for someone who is critically ill. ${ }^{22}$ This agrees in part with the findings of Van den Berghe et al, ie, that it may be an acute fluctuation in blood glucose that is most damaging. ${ }^{5}$ Many studies investigating the effect of blood glucose control are retrospective, and can only derive whatever information was recorded at the time, and in most cases significant details are not available. ${ }^{22}$ Several commentators, including Alves et al, have expressed frustration at the lack of conclusive evidence for or against tight glycemic control in children after surgery due to their "premature physiology", the variety of "surgical procedures" to which they can be exposed, and "less uniformity in preoperative conditions". 9

Alves et $\mathrm{al}^{9}$ found a statistically significant difference between mean blood glucose in the first 24 hours and postoperative complications, compared with Polito et a ${ }^{25}$ who found that outcomes were insignificant until 72 hours after surgery. ${ }^{25}$ One study investigated the incidence of mediastinitis following median sternotomy; a procedure that has a mortality rate of $6 \%-23 \%{ }^{26}$ These patients may have a prolonged hospital stay and may require extended antibiotic therapy, but Ghafoori et al showed that persistent postoperative hyperglycemia was more predictive of adverse outcomes than the initial postoperative blood glucose value. ${ }^{26} \mathrm{~A}$ key finding of Alves et al was the importance of exogenous corticosteroid administration and the use of extracorporeal 
circulation as two specific risk factors for development of secondary complications. ${ }^{9}$ Postoperative hyperglycemia is all-encompassing according to Ulate et al, and the intensity, duration and variability are all associated with poor outcomes in critically ill children. ${ }^{8}$ Hanazaki and Okabayashi ${ }^{27}$ suggested that a critical cutoff of $140 \mathrm{mg} / \mathrm{dL}$ is necessary for the prevention of surgical site infections and Ata et al reported that perioperative hyperglycemia is the most important risk factor for post-surgical infection. ${ }^{28} \mathrm{Wu}$ et al also found a correlation between those children with the highest rates of hyperglycemia (>200 mg/dL) and infection risk, although this was not significant and does not rule out other disturbances which predispose these children to infection. ${ }^{29}$

\section{Pediatric glycemia}

It is estimated that 20,000 operations are carried out each year in the US for the correction of congenital heart defects. ${ }^{1}$ The incidence of hyperglycemia, defined as $>126 \mathrm{mg} / \mathrm{dL}$, is estimated to be up to $90 \%$ in these patients, but the correction of which, risks iatrogenic hypoglycemia that may cause damage to the brain. ${ }^{1}$ Children have a higher recommended optimal blood glucose level of $110-126 \mathrm{mg} / \mathrm{dL}$, which is higher than in adults, ${ }^{30}$ and levels $>140 \mathrm{mg} / \mathrm{dL}$ can be classed as hyperglycemia. ${ }^{22}$ The perioperative period is an extremely unusual period of time for the body characterized by extremes in blood volume, body temperature, plasma composition, and tissue blood flow, as reported by Alves et al. ${ }^{9}$ Further, Filho et al stress the importance of selecting an appropriate target group because the consequences of any fluctuations in blood glucose depend on the intervention performed and preoperative morbidity. ${ }^{10}$

Fundamental differences in the physiology of a child compared with that of an adult do not permit us to extrapolate findings from adult studies to that of children, and as such, certain considerations must be met. The effects of hypoglycemia on a developing brain are characterized by damage to many brain structures, including both gray and white matter, the hippocampus, basal ganglia, hypothalamus, and brain stem, ${ }^{3}$ and for this reason, it may seem logical to advocate a "wait and see" approach when the secondary effects of moderate hyperglycemia are not fully known. Children have a relatively reduced glycogen reserve compared with adults, especially when under stress or given insulin. ${ }^{23}$ As reported by Hirshberg et al, ${ }^{20}$ the fear of inducing hypoglycemia in children seems to outweigh any potential benefit of reducing hyperglycemic levels, but it could be the case that erring on the side of caution may be causing harm. The risk of hypoglycemia is further compounded by the findings of Calabria et al who, in their study investigating "dumping syndrome" in children following fundoplasty, reported that up to $50 \%$ of at-risk infants had hypoglycemic episodes without displaying any symptoms. ${ }^{6}$ Subtle differences between the physiology of children and adults may create significantly different outcomes.

It is particularly challenging to manage children before surgery because withholding glucose can induce hypoglycemia and children can still be susceptible to surgery-related hyperglycemia. ${ }^{3}$ However, research has shown that normal healthy infants can withstand prolonged fasting and it may not be necessary to administer preoperative glucose or to even monitor glucose in these patients. ${ }^{31}$ In 2012, a NICE-SUGAR follow-up trial identified a significant relationship between hypoglycemia and mortality, but could not prove causality. ${ }^{32}$ Importantly, the authors concluded that the severity of hypoglycemia at day 1 was the most important predictor of mortality in their large-scale prospective trial of 6,026 patients. ${ }^{32}$ In conclusion, the authors recommended a target blood glucose of $144-180 \mathrm{mg} / \mathrm{dL}$ to avoid the dangerous complications of hypoglycemia in critically ill adult patients. ${ }^{32}$

\section{Neonatal hypoglycemia}

Neonates are a unique group of patients in whom the effects of both hypoglycemia and hyperglycemia can be pronounced. Ballweg et al, in a study of 188 children aged $<6$ months, suggested that hyperglycemia may be neuroprotective due to the enhanced clearance of lactate, but hypoglycemia can cause lasting neurologic damage if levels fall below $40 \mathrm{mg} / \mathrm{dL} .{ }^{33}$ Further, it was suggested that glucose may protect oligodendrocytes and glial cells from hypoxia-induced periventricular leukomalacia. ${ }^{33}$ The prevalence of neonatal hypoglycemia is high at $5 \%-15 \%$ in otherwise healthy babies. ${ }^{34}$ Due to the unacceptability of repeated invasive blood glucose monitoring, Harris et al performed a short-term cross-sectional study on 514 neonates aged $<48$ hours who were considered to be at risk for hypoglycemia due to maternal diabetes, being late-preterm, or small or large at birth. ${ }^{34}$ Seventy-nine percent of babies became hypoglycemic, $15 \%$ were too sleepy to feed when hypoglycemic, and only $7 \%$ had visible signs. ${ }^{34}$ Any neonate experiencing an episode of hypoglycemia was fed with dextrose gel to correct this, but the study stressed the importance of hypervigilance in this vulnerable patient population. ${ }^{34}$

\section{Design flaws}

Difficulties arise with the creation of a standardized approach to quantify the effect of blood glucose on outcomes. Some studies utilize a multifactorial approach whereas 
others stress the importance of one over the other. Another key factor is the recruitment of an appropriate patient group, which is especially important in pediatric studies; some studies define "pediatric" as ranging from 0 to 18 years of age, whereas others exclusively recruit children from a narrow age group such as $0-36$ months. ${ }^{1}$ Further, a wide range of surgical procedures of differing degrees of invasiveness and interindividual variations, such as preoperative comorbidities, ethnicity, age, surgical proficiency, and standard of aftercare, mean it is extremely difficult to extrapolate results to a wider population. Typically, bedside glucose meters are used to measure blood glucose levels but these are often inaccurate and tend to overestimate true plasma glucose levels. ${ }^{7}$ In fact, point-of-care monitors give readings typically $10 \%$ lower. ${ }^{22}$ Further, Steven and Nicolson remark that bedside meters are subject to many other variables, including hematocrit, hypothermia, and hypotension. ${ }^{3}$ The difficulty in designing a study which could inform clinical practice is hampered by a lack of consistency in screening and treatment strategies between centers. ${ }^{8}$ Pediatric cardiac surgery has an expected mortality of $3 \%-5 \%$, and although there is an abundance of children undergoing such procedures, small studies cannot generate sufficient power to provide any authority on the effect of glucose control on mortality, which many would argue is the key outcome of any intervention..$^{30}$ Other studies combine outcomes, such as infection and mortality, which in practice are not similar in their severity or threat to life. ${ }^{3}$

\section{A permissible glycemic target?}

The NICE-SUGAR trial in adult patients suggested that a moderate level of hyperglycemia may form a trade-off between the risks of severe hyperglycemia and the danger of iatrogenic hypoglycemia. ${ }^{18}$ It is difficult to define the level at which glucose levels become dangerous, but it is thought that levels $>200 \mathrm{mg} / \mathrm{dL}$ are associated with increased morbidity and mortality; however, the same effect can be seen at lower levels, such as $140 \mathrm{mg} / \mathrm{dL}$, depending on the individual. ${ }^{3}$ Small studies have shown that a more "liberal" target may be beneficial and may appease clinicians who are concerned about regulating blood glucose too tightly in pediatric patients. ${ }^{8,35}$ To address this issue, Ulate et al conducted a retrospective, case-controlled study to try and determine to what extent "hyperglycemia" would be acceptable. ${ }^{35}$ Overall, they concluded that, in the 177 patients investigated, a permissible target could improve secondary outcomes and could decrease episodes of hypoglycemia within 5 days. ${ }^{35}$ Mortality rates were actually lowest in the permissive target group (4.6\%) compared with the euglycemia group (6.02\%), the moderate hyperglycemia group (38.8\%), and the severe hyperglycemia group $(58.3 \%){ }^{35}$ This was certainly a positive finding worthy of further consideration, but the study itself was retrospective, low-powered, and used unreliable measures of glucose levels. ${ }^{35}$ Ulate et al suggested that a safe cutoff for hypoglycemia would be $<60 \mathrm{mg} / \mathrm{dL}$ because of the neurocognitive deficits observed at levels below this. ${ }^{35}$ Regrettably, it is likely that the more severely ill children would have had more regular blood glucose measurements taken, so we cannot state whether these values accurately represent the sample population. ${ }^{29,35}$ Further, several aspects of care are carried out at the discretion of the physician, including administration of insulin, which makes it extremely difficult to determine what constitutes "good practice" and formulate guidelines accordingly, or to interpret the outcome of interventions that are not exhaustively documented. ${ }^{29,35}$ This study did not investigate the method of postoperative feeding, something which Marik and Preiser emphasized as being crucially important. . $^{735}$

\section{Treating hypoglycemia}

Rates of presurgical hypoglycemia in children are thought to be between $0.2 \%$ and $2.5 \%,{ }^{3}$ with neonates at most risk due to their inability to respond metabolically. ${ }^{3}$ To counteract this, it became widely accepted practice to administer dextrose, which led to hyperglycemia. ${ }^{3}$ During surgery, fluid replacement aims to provide basal energy requirements and compensates any fasting deficit; unfortunately, Murat and DuBois believe that guidelines are not always applicable or followed. ${ }^{31}$ This suggests that routine intraoperative solution use should potentially be re-evaluated in terms of secondary post-surgical outcomes. ${ }^{31}$

\section{Traumatic brain injury in children}

Studies in adults have shown that adults exposed to a traumatic brain injury are particularly at risk of hyperglycemia. ${ }^{14}$ The same can be said of children, because they are particularly susceptible to the detrimental effects of hyperglycemia. Hyperglycemia is one of many factors responsible for increased mortality in this patient cohort; others include hypoxia, brain damage, raised intracranial pressure, and hypotension. ${ }^{36}$ In conditions such as this, where time is key, an early marker of severity is the level of hyperglycemia found on admission and within the first 24 hours. ${ }^{36}$ The reason why hyperglycemia is so pronounced following trauma, burns, and severe injuries is the induction of a hypermetabolic state. ${ }^{37}$ Many studies investigating 
traumatic brain injury are retrospective, and as such, it is difficult to find articles in which a well defined glucose testing schedule was used. ${ }^{36}$

Sharma et al reported that, of 107 patients, only 64\% had hourly glucose measurements. ${ }^{36}$ Their study was wide in scope because they wanted to address whether preoperative, intraoperative, and/or postoperative hyperglycemia had the biggest effect on outcome for children undergoing craniotomy. ${ }^{36}$ Due to the paucity of measurements, their results were not scientifically robust and no conclusions could be drawn accurately. ${ }^{36}$ This study did propose the usefulness of a continuous glucose monitor, something which Steil et al assessed 2 years later. ${ }^{21,36}$ Further, due to the nature of the study, long-term follow-up was not possible, something that is of utmost importance following a severe brain injury.

\section{Cardiac surgery and cardiopulmonary bypass (CPB)}

As well as providing a significant number of patients per year for analysis, cardiac surgery is also particularly interesting in the context of hyperglycemia since extracorporeal circulation consistently causes hyperglycemia. ${ }^{3}$ Difficulty arises when we attempt to quantify hyperglycemia or indeed hypoglycemia, and patients, especially newborns or those under sedation, may not exhibit any symptoms until their blood glucose level has fallen dangerously low. ${ }^{3}$ A meta-analysis carried out by $\mathrm{Ng}$ et al found that tight blood glucose control caused hypoglycemia in one of four RCTs studied, but due to discrepancies in the definition of hypoglycemia and a lack of data concerning other secondary cardiovascular outcomes, they stress the need for further study. ${ }^{38} \mathrm{~A}$ major factor in the generation of intraoperative hyperglycemia is glucocorticoid administration. ${ }^{9,37}$

Opinion differs on the optimum time to intervene following cardiopulmonary bypass. DeCampli et al found that levels are highest immediately after cardiopulmonary bypass and tend to decline steadily over the next 2 days, but that this effect depended largely on the concentration of corticosteroids used. ${ }^{13}$ Cardiopulmonary bypass is associated with characteristic hormonal mechanisms which predispose the individual to hyperglycemia, including an increase in growth hormone and insulin production. ${ }^{37}$ However, Verhoven et al argue that routine administration of glucocorticoids prior to cardiopulmonary bypass to reduce the mild inflammatory response seen post-cardiac surgery is not evidence-based and instead forms part of an historic approach which may now be considered outdated. ${ }^{39}$ Above all, Verhoven et al suggest that the decision to treat should be based on pathophysiology and any intervention should be closely scrutinized. ${ }^{39}$ This opinion was echoed by Yates et al, who found that glucose levels did not increase "as much as expected" following the administration of glucocorticoids, and attributed the modest rise to cardiopulmonary bypass alone. ${ }^{40}$ Generally speaking, adults typically show hyperglycemia with raised insulin levels compared with children who have low insulin levels. ${ }^{40}$ The insulin resistance seen in children following cardiopulmonary bypass could be due to a variety of factors, including beta-cell dysfunction, post-surgical hypothermia, or use of vasopressors. $^{40}$

\section{Conclusion}

The myriad of small-powered studies makes it impossible to argue convincingly one way or the other whether tight glucose management is of benefit in either adults or children. Results have been so varied that the American College of Physicians has actively recommended against the use of intensive insulin therapy in children. ${ }^{41}$ Disagreements between two major studies investigating tight glucose management in adults has led to unclear objectives, and in many ways has raised more questions than it has solved. A distinct lack of well designed, prospective RCTs means research has many unanswered questions. In children, where the effects of fluctuating blood glucose levels are not fully understood, it may well be the case that research has ground to a halt. There is simply not enough evidence to suggest that tight glycemic control would be of benefit in this patient population because the risks of hypoglycemia are felt to be too great. Until long-term data either confirming or refuting intensive insulin therapy in this population of patients is found, current practice is unlikely to change. Further, the ethical implications of a randomized blinded trial which risks creating significant disability in children whose neurological systems are immature are unacceptable. On the other hand, being too cautious and exposing children to hyperglycemia of any intensity may come with its own risks. It is highly likely that glycemic targets will be of most benefit if they are age-related and take into account individual variability.

\section{Disclosure}

Neil Christopher Forbes is a final year medical student at Manchester University, UK. He was previously employed at Dove Medical Press, Macclesfield, UK. He has also acted as a part-time commissioning editor for Dove Medical Press journals, although not for the International Journal of General Medicine. 


\section{References}

1. Agus MS, Steil GM, Wypij D, et al. Tight glycemic control versus standard care after pediatric cardiac surgery. $N$ Engl J Med. 2012;367:1208-1218

2. Vanhorebeek I, Langouche L. Molecular mechanisms behind clinical benefits of intensive insulin therapy during critical illness: glucose versus insulin. Best Pract Res Clin Anaesthesiol. 2009;23:449-459.

3. Steven J, Nicolson S. Perioperative management of blood glucose during open heart surgery in infants and children. Pediatr Anaesth. 2011;21:530-537.

4. Van den Berghe G, Wouters P, Weekers F, et al. Intensive insulin therapy in critically ill patients. $N$ Engl J Med. 2001;345:1359-1367.

5. Van den Berghe G, Schetz M, Vlasselaers D, et al. Intensive insulin therapy in critically ill patients: NICE-SUGAR or Leuven blood glucose target? J Clin Endocrinol Metab. 2009;94:3163-3170.

6. Calabria AC, Gallagher PR, Simmons R, Blinman T, De Leon DD. Postoperative surveillance and detection of postprandial hypoglycemia after fundoplasty in children. J Pediatr. 2011;159:597-601.

7. Marik PE, Preiser JC. Toward understanding tight glycemic control in the ICU. Chest. 2010;137:544-551.

8. Ulate KP, Raj S, Rotta AT. Critical illness hyperglycemia in pediatric cardiac surgery. J Diabetes Sci Technol. 2012;6:29-36.

9. Alves RL, Cerqueira MP, de Castro Kraychete NC, et al. Perioperative blood glucose level and postoperative complications in pediatric cardiac surgery. Arq Bras Cardiol. 2011;97:372-379.

10. Filho EM, de Carvalho WB, Cavalheiro S, et al. Hyperglycemia and postoperative outcomes in pediatric neurosurgery. Clinics. 2011;66:1637-1640.

11. Van den Berghe G, Wilmer A, Hermans G, et al. Intensive insulin therapy in the medical ICU. $N$ Engl J Med. 2006;354:449-461.

12. De La Rosa GDC, Donado JH, Restrepo AH, et al. Strict glycemic control in patients hospitalised in a mixed medical and surgical intensive care unit: a randomised clinical trial. Crit Care. 2008;12:1-9.

13. DeCampli WM, Olsen MC, Munro HM, Felix DE. Perioperative hyperglycemia: effect on outcome after infant congenital heart surgery. Ann Thorac Surg. 2010;89:181-186.

14. Meng Y, Qingjie G, Xiangtong Z, et al. Intensive insulin therapy on infection rate, days in NICU, in-hospital mortality and neurological outcome in severe traumatic brain injury patients: a randomised controlled trial. Int J Nurs Stud. 2009;46:7532-7758.

15. Verbruggen SC, de Betue CT, Schierbeek H, et al. Reducing glucose infusion safely prevents hyperglycemia in post-surgical children. Clin Nutr. 2011;30:786-792.

16. Gandhi GY, Nuttall GA, Abel MD, et al. Intraoperative hyperglycemia and perioperative outcomes in cardiac surgery patients. Mayo Clin Proc. 2005;80:862-866.

17. Hua J, Chen $\mathrm{G}, \mathrm{Li} \mathrm{H}$, et al. Intensive intraoperative insulin therapy versus conventional insulin therapy during cardiac surgery: a meta-analysis. J Cardiothorac Vasc Anesth. 2012;26:829-834.

18. The NICE-SUGAR Study Investigators. Intensive versus conventional glucose control in critically ill patients. $N$ Engl J Med. 2009;360: 1283-1297.

19. Vlasselaers D, Milants I, Desmet L, et al. Intensive insulin therapy for patients in pediatric intensive care: a prospective, randomised controlled study. Lancet. 2009;373:547-556.

20. Hirshberg E, Lacriox J, Sward K, Wilson D, Morris AH. Blood glucose control in critically ill adults and children. Chest. 2008;133: 1328-1335.

21. Steil GM, Langer M, Jaeger K, et al. Value of continuous glucose monitoring for minimising severe hypoglycemia during tight glycemic control. Pediatr Crit Care Med. 2011;12:643-648.
22. Van den Berghe G, Vlasselaers D, Desmet L, Vanhorebeek I, Mesotten D. Glycemic control in pediatric critical care. Lancet. 2009;373:1423-1424.

23. Piper HG, Alexander JL, Shukla A, et al. Real-time continuous glucose monitoring in pediatric patients during and after cardiac surgery. Pediatrics. 2006;118:1176-1186.

24. O'Brien JE, Marshall JA, Tarrants ML, Stroup RE, Lofland GK. Intraoperative hyperglycemia and postoperative bacteremia in the pediatric cardiac surgery patient. Ann Thorac Surg. 2010;89:578-584.

25. Polito A, Thiagarajan RR, Laussen PC, et al. Association between intraoperative and early postoperative glucose levels and adverse outcomes after complex congenital heart surgery. Circulation. 2008; 118:2235-2242.

26. Ghafoori AF, Twite MD, Friesen RH. Postoperative hyperglycemia is associated with mediastinitis following pediatric cardiac surgery. Pediatr Anaesth. 2008;18:1202-1207.

27. Hanazaki K, Okabayashi T. What should the targeted range of blood glucose levels be to reduce the incidence of surgical site infection following general surgery? Arch Surg. 2011;146:368-369.

28. Ata A, Lee J, Bestle SL, Desemone J, Stain SC. Postoperative hyperglycemia and surgical site infection in general surgery patients. Arch Surg. 2010;145(9): 858-864.

29. Wu Y, Pei J, Yang XD, et al. Hyperglycemia and its association with clinical outcomes for patients in the pediatric intensive care unit after abdominal surgery. J Pediatr Surg. 2013;48:801-805.

30. Gaies MG, Langer M, Alexander J, et al. Design and rationale of safe pediatric euglycemia after cardiac surgery: a randomized controlled trial of tight glycemic control after pediatric surgery. Pediatr Crit Care Med. 2013;14:148-156.

31. Murat I, DuBois MC. Perioperative fluid therapy in pediatrics. Pediatr Anaesth. 2008;18:363-370.

32. The NICE-SUGAR Study Investigators. Hypoglycemia and risk of death in critically ill patients. $N$ Engl J Med. 2012;367:1108-1118.

34. Harris DL, Weston PJ, Harding JE. Incidence of neonatal hypoglycemia in babies identified as at risk. J Pediatr. 2012;161:787-791.

33. Ballweg JA, Wernovsky G, Ittenbach RF, et al. Hyperglycemia after infant cardiac surgery does not adversely impact neurodevelopmental outcome. Ann Thorac Surg. 2007;84:2052-2058.

35. Ulate KP, Lima Falcao GC, Bielefeld MR, Morales JM, Rotta AT. Strict glycemic targets need not be so strict: a more permissive glycemic range for critically ill children. Pediatrics. 2008;122:e898-e904.

36. Sharma D, Jelacic J, Chennuri R, Chaiwat O, Chandler W. Incidence and risk factors for perioperative hyperglycemia in children with traumatic brain injury. Anesth Analg. 2009;108:81-89.

37. Alaei F, Davari PN, Alaei M, Azarfarin R, Soleymani E. Postoperative outcome for hyperglycemic pediatric cardiac surgery patients. Pediatr Cardiol. 2012;33:21-26.

38. $\mathrm{Ng} \mathrm{K}$, Grounds R, Haga K, Carter G, Clarke S. The efficacy and safety of tight blood glucose control during heart surgery: a systematic review and meta-analysis. Anaesthesia. 2009;64:1387-1389.

39. Verhoven JJ, Hokken-Koelega AC, den Brinker M, et al. Disturbance of glucose homeostasis after pediatric cardiac surgery. Pediatr Cardiol. 2011;32:131-138.

40. Yates AR, Dyke PC 2nd, Taeed R, et al. Hyperglycemia is a marker for poor outcome in the postoperative pediatric cardiac patient. Pediatr Crit Care Med. 2006; 7:351-355.

41. Srinivasan V. Examining the 'SPECS' of the Safe Pediatric Euglycemia After Cardiac Surgery Study: thinking outside the box for safer control of blood glucose in the ICU. Pediatr Crit Care Med. 2013;14:226-227. 
International Journal of General Medicine

Dovepress

\section{Publish your work in this journal}

The International Journal of General Medicine is an international, peer-reviewed open-access journal that focuses on general and internal medicine, pathogenesis, epidemiology, diagnosis, monitoring and treatment protocols. The journal is characterized by the rapid reporting of reviews, original research and clinical studies across all disease areas.
A key focus is the elucidation of disease processes and management protocols resulting in improved outcomes for the patient. The manuscript management system is completely online and includes a very quick and fair peer-review system. Visit http://www.dovepress.com/ testimonials.php to read real quotes from published authors.

Submit your manuscript here: http://www.dovepress.com/international-journal-of-general-medicine-journal 